\title{
Effect of compelled body weight shift therapy on weight bearing symmetry and balance in post stroke patients: an experimental pre-post study
}

\section{JEBA CHITRA AND SIDDHARTH MISHRA}

Author for Correspondence : SIDDHARTH MISRA

KLEU Institute of Physiotherapy, JNMC

Campus, BELGAUM (KARNATAKA) INDIA

\section{KEY WORDS :}

Compelled body weight shift therapy, Stroke, Weight bearing symmetry, Balance; Berg balance scale

\begin{abstract}
:
Introduction:Stroke related hemiparesis also exhibit asymmetry in standing posture and walking. Chronic stroke survivors represent with asymmetric gait pattern due to altered weight distribution.Altered weight distribution of lower limbs that is less weight is taken on affected side, less excursion on the weaker side, is seen during static and dynamic balance as well as external perturbation.

Objective:To evaluate the effect of Compelled Body Weight Shift Therapy on weight bearing symmetry and balance in post stroke patients.

Methods: Total 22 participants were included in the study. All patients received $10 \mathrm{~mm}$ shoe insole which is to be used on their unaffected side and along with this conventional rehabilitation program were given for 1 hour per day for 2 weeks.

Results: There was significant difference in pre-post weight bearing distribution $(\mathrm{p}<0.001)$ and balance $(\mathrm{p}<0.001)$. There was also significant difference in all the component of Berg Balance Scale $(\mathrm{p}<0.001)$.

Conclusion: Compelled Body Weight Shift Therapy can be easily administrated in the daily rehabilitation protocol, while treating stroke patients with balance problems due to asymmetrical weight bearing. With more symmetrical weight bearing, balance can be improved for better ambulation and reduced risk of falls.
\end{abstract}

How to cite this paper : Chitra, Jeba and Mishra, Siddharth (2014). Effect of compelled body weight shift therapy on weight bearing symmetry and balance in post stroke patients: an experimental pre-post study. Internat. J. Med. Sci., 7(1\&2) : 12-17.
Paper History : 